\title{
BMJ Open How do healthcare providers construe patient complexity? A qualitative study of multimorbidity in HIV outpatient clinical practice
}

\author{
Shiko Ben-Menahem (D) , ${ }^{1}$ Anastassja Sialm, ${ }^{1}$ Anna Hachfeld, ${ }^{2}$ Andri Rauch, ${ }^{2}$ \\ Georg von Krogh, ${ }^{1}$ Hansjakob Furrer ${ }^{2}$
}

To cite: Ben-Menahem S, Sialm A, Hachfeld A, et al. How do healthcare providers construe patient complexity? A qualitative study of multimorbidity in HIV outpatient clinical practice. BMJ Open 2021;11:e051013. doi:10.1136/ bmjopen-2021-051013

- Prepublication history and additional supplemental material for this paper are available online. To view these files, please visit the journal online (http://dx.doi.org/10.1136/ bmjopen-2021-051013).

Received 08 March 2021 Accepted 29 October 2021

Check for updates

(c) Author(s) (or their employer(s)) 2021. Re-use permitted under CC BY-NC. No commercial re-use. See rights and permissions. Published by BMJ.

${ }^{1}$ Department of Management, Technology and Economics, ETH Zurich, Zurich, Switzerland

${ }^{2}$ Department of Infectious Diseases, Inselspital University Hospital Bern, Bern, Switzerland

Correspondence to Dr Shiko Ben-Menahem; benmenahem@ethz.ch

\section{ABSTRACT}

Introduction Patient complexity is an increasingly used concept in clinical practice, policy debates and medical research. Yet the literature lacks a clear definition of its meaning and drivers from the health provider's perspective. This shortcoming is problematic for clinical practice and medical education in the light of a rising number of multimorbid patients and the need for future healthcare providers that are adequately trained in treating complex patients.

Objectives To develop an empirically grounded framework of healthcare providers' perceptions of patient complexity and to characterise the relationship between case complexity, care complexity and provider experience as complexity-contributing factors.

Design Qualitative study based on semistructured indepth interviews with healthcare practitioners.

Setting A Swiss hospital-based HIV outpatient clinic. Participants A total of 31 healthcare providers participated. Participants volunteered to take part and comprised 17 nurses, 8 junior physicians (interns) and 6 senior physicians (residents, fellows and attendings). Results Perceived patient complexity arises from the combination of case complexity drivers, the provider's perceived controllability, and a set of complexity moderators at the levels of the patient, the care provider and the broader care context. We develop a conceptual framework that outlines key relationships among these complexity-contributing factors and present 10 key questions to help guide medical professionals in making complexity more explicit and more manageable in daily practice.

Conclusions The framework presented in this study helps to advance a shared understanding of patient complexity. Our findings inform curriculum design and the teaching of essential skills to medical students in areas characterised by high patient complexity such as general internal medicine and geriatrics. From a policy perspective, our findings have important implications for the design of more effective healthcare interventions for complex patients.

\section{INTRODUCTION}

Providing medical care for complex patients constitutes one of the most challenging aspects of modern healthcare systems. In clinical practice and the research literature,

\section{STRENGTHS AND LIMITATIONS OF THIS STUDY}

$\Rightarrow$ Primary data on healthcare providers' perceptions of patient complexity.

$\Rightarrow$ Data sourced across professions (physicians, nurses) and experience levels.

$\Rightarrow$ The study reports findings from a single outpatient setting. Results may not be generalisable to other primary care settings.

$\Rightarrow$ The study only differentiates between two levels of experience (junior and senior healthcare providers).

the concept 'complex patient' typically refers to patients with coexisting chronic conditions (ie, comorbidity, multimorbidity, polypathology, dual diagnosis) and challenges associated with managing interactions among various conditions and medications. Medical complexity-the number of comorbiditiesposes well-known challenges for patients, healthcare professionals and healthcare systems. Yet studies investigating complex patients show that while the degree of comorbidity is informative for gauging the degree to which physicians experience a patient as 'complex', such measures do not fully capture complexity from the health provider's perspective. $^{1-6}$ Indeed, practitioners' understanding of complexity entail a much broader set of contributing factors, including the patient's psychosocial vulnerabilities, socioeconomic environment, cultural background and behavioural factors. ${ }^{2578}$

While the importance of incorporating a broad range of complexity-contributing factors into research on complex patients is increasingly recognised, recent studies indicate that much remains to be understood about patient complexity in clinical settings. Indeed, an analysis of the health science literature between 2005 and 2015 found that 'the situational, relational, temporal, sociocultural 
and clinical contexts in which the concept (of patient complexity) is relevant, effectively used and applied in various situations has not...been critically examined in health sciences' and that 'an in-depth analysis of complexity itself and what it means in clinical practice' is lacking in the literature. ${ }^{9}$ The authors accordingly call for in-depth qualitative research about patient complexity and the ways in which medical practitioners experience it.

Building on literature showing the importance of studying patient complexity from the clinician's perspective and studies calling for a broad understanding of patient complexity beyond number of conditions and medications, ${ }^{239}$ this study develops empirically grounded knowledge on how complexity-contributing factors of HIV patients translate into healthcare providers' perceptions of care complexity in a hospital based outpatient clinic for infectious diseases (ID). Understanding the meaning, drivers and outcomes of patient complexity from the healthcare provider's perspective and the role of experience in complexity perceptions is of significant practical relevance. In HIV clinical care, for example, advances in antiretroviral therapies, disease screening and health promotion have significantly improved the life expectancy of HIV-positive individuals. ${ }^{10}$ Consequently, age-related multimorbidity pose new, hence incompletely understood challenges for clinicians and healthcare planners, challenges that require medical education, effective healthcare interventions and organisational support systems. $^{81112}$ For example, care for complex patients involves close coordination among specialists forming multidisciplinary teams. Ensuring that such teams achieve positive patient outcomes requires that care providers collectively understand the nature of patient complexity. From an education and policy perspective, developing a better concept of perceived complexity and the role of the practitioner's experience is critical for designing effective healthcare training and interventions that improve patient care while curbing the disproportional use of healthcare resources for complex patients.

\section{Study overview}

To elaborate our understanding of care providers' complexity perceptions, we conducted a qualitative study based on semistructured in-depth interviews with healthcare practitioners with different levels of experience. Our data collection and analysis builds on a separation of two domains (This conceptualisation reflects the separation of case complexity and care complexity (de Jonge et al; Doessing and Burau) on one hand, and the separation of patient complexity and clinical task complexity (Islam $e t$ $a l)$ on the other hand.) of complexity in HIV clinical practice: (1) patient-related factors (case complexity) and (2) care delivery-related factors (care complexity). ${ }^{71314} \mathrm{We}$ advance prior work on patient complexity by exploring under what conditions case complexity translates into perceived care complexity. Focusing on complexity perceptions among nurses, junior physicians and senior physicians, we also address the paucity in research on differences in complexity perceptions among healthcare professions and among healthcare providers with different levels of experience.

\section{METHODS}

To develop understanding of healthcare providers' perceptions of patient complexity and the role of experience therein, we conducted a qualitative study of practitioners across medical professions (ie, nurses and physicians) with varying levels of experience and (ie, junior and senior). Within the qualitative paradigm, we conducted a phenomenological approach, so as to develop an in-depth understanding of patient complexity perceptions within the broader social context of the medical practitioner's work setting. ${ }^{15-17}$ This approach allows the researcher the focus on practitioners' lived experiences with respect to patient complexity and the issues influencing the construction of individual perceptions of complexity. Interpretative phenomenological analysis (IPA) provided the general research framework for our data collection and data analysis process. ${ }^{18-20}$ IPA foregrounds that the meanings that individuals attribute to their experiences can be accessed and understood through an interpretative process that focuses on the subject's individual cognitive inner world.

\section{Patient and public involvement}

No patients were involved in this study.

\section{Participants and setting}

We conducted a qualitative study using semistructured interviews with nurses and junior and senior physicians of an ID department in a high-capacity Swiss university hospital, focusing on the department's HIV outpatient care activities. In collaboration with the clinic director, we scheduled interviews with all available department members, resulting in 31 participants, including 7 senior nurses, 10 junior (assistant) nurses, 8 junior physicians (interns) and 6 senior physicians (residents, fellows and attendings). The average professional experience of study participants was 15.62 years. Seventy-four per cent of participants was female. Department members were asked to participate in the study and were free to decline. One person declined to participate citing time constraints. Verbal informed consent was obtained (and voice recorded) from each participant after explaining the study procedures and data use before starting the interview. Patients or the public were not involved in the design, or conduct, or reporting, or dissemination plans of our research.

\section{Study materials}

To structure our interviews, we used an interview guide focusing on healthcare providers' perceptions of patient complexity in both multimorbid and non-multimorbid patients, with a focus on the department's activities in HIV clinical practice. In phase 1 of our study, we developed our 
interview guide (see online supplemental appendix I) in two stages. In the first stage, two members of the research team, the principal investigator for this study (SB-M) and a research assistant (AS), conducted exploratory pilot interviews with department members, including senior and junior nurses and senior and junior physicians. The questions in this exploratory round were based on our review of the literature on coordination of care for multimorbid patients and 2 days of observations of the clinical setting. Interviews lasted between 45 and $60 \mathrm{~min}$. From these interviews and a further analysis of the literature on complex patients, we reached consensus on an initial set of questions covering the healthcare provider's background and daily activities, perceptions of complexity and practices and strategies used in treating complex patients. In the second stage, we conducted a roundtable discussion with 12 department members. During this session which we (1) asked participants to broadly reflect on their perceptions of patient complexity and its contributing factors in the context of HIV clinical practice and (2) check our initial set of questions for clarity. The purpose of this session was to refine our interview guide and ensure questions were relevant to the research context.

\section{Data collection}

Using the insights from phase 1, in phase 2 of the study, SB-M and AS conducted 31 semistructured interviews over a 6-month period. Participants were briefed on the purpose and confidentiality of the interviews, and were encouraged to share detailed personal experiences as much as possible. Interviews took place in physicians' offices or other private spaces chosen by the interviewees. Interviews lasted around $60 \mathrm{~min}$ on average, and were digitally recorded and professionally transcribed verbatim.

\section{Data analysis}

The final data consisted of 320 pages of single-spaced transcripts. Transcribed files were stored and thematically analysed in NVivo V.11, a qualitative data analysis software (QSR International). We followed a systematic inductive procedure for analysing qualitative data. ${ }^{21}$ First, all transcripts (raw data) were closely read multiple times by SB-M and AS. During this stage, we discussed the meaning of interview segments to develop an initial coding scheme. Using the initial version of the coding scheme, we double blindly coded a random selection of 10 interview transcripts. We next discussed disparately coded segments and refined the coding scheme in several rounds. After reaching consensus on the coding scheme, we divided the transcripts between SB-M and AS and coded all transcripts according to the new coding scheme. Remaining ambiguities in the data were jointly resolved. This inductive approach allowed for overarching general categories and more specific lower-level categories on complexity perceptions and relations among these categories to emerge from the data. We also paid attention to differences and similarities in complexity and controllability perceptions across levels of experience. While experience in years of relevant ranged from recent graduates to 34 years, we applied a binary distinction (junior vs senior) in the presentation of our results, where junior refers to nurses and physicians with less than 3 years of experience since graduation. Perceived controllability was categorised in three levels (low, medium, high) and reflect professionals' experienced ability to diagnose the patient, and identify and execute treatment options as a result of specific complexitycontributing factors. We defined low controllability as cases in which professionals reported to be significantly constrained in their ability to coordinate and deliver patient care; medium controllability as cases in which professionals reported to be somewhat limited in their ability to do so, and high controllability as cases in which professionals reported to experience no limitations resulting from complexitycontributing factors.

From this initial analysis, we developed a preliminary framework of the main domains and drivers of complexity perceptions among healthcare providers. In producing the final framework and reporting the study, SB-M, AS and GvK elicited and incorporated the perspectives of three participants $(\mathrm{AH}$, who is an attending physician in ID, AR, who is a professor of ID, and HF, who is head of department and professor of ID) to promote trustworthiness of the study. The perspectives of the three participants were incorporated through informal discussions and multiple rounds of feedback on the manuscript, and covered the interpretation of raw data, the grouping of themes, the emergent framework. Member checking with these participant-authors thus served to confirm the validity of our findings and ensure sensitivity to the research context.

\section{RESULTS}

Figure 1 shows a conceptual framework of our key findings. Our analysis revealed how case complexity drivers and a set of complexity moderators at the patient level, care provider level and care context level jointly determined care providers' perceived controllability-referring to the provider's sense of their ability to diagnose and exercise control over the patient's health state. Providers' perceived controllability, in turn, was a key driver of perceived care complexity. We next elaborate on these findings.

\section{Case complexity drivers}

Participants described complexity-contributing factors relating to the patient's medical health state as the primary component of case complexity. Factors in this category included multimorbidity and polypharmacy, mental health and changes in the patient's health state.

\section{Multimorbidity, polypharmacy and instability}

Participants identified the presence of multiple chronic medical conditions as a general driver of perceived patient complexity. As one junior physician explained:

A simple situation would be a patient who needs his HIV drugs and takes them regularly and has no contraindication to receiving a single tablet regimen. A 


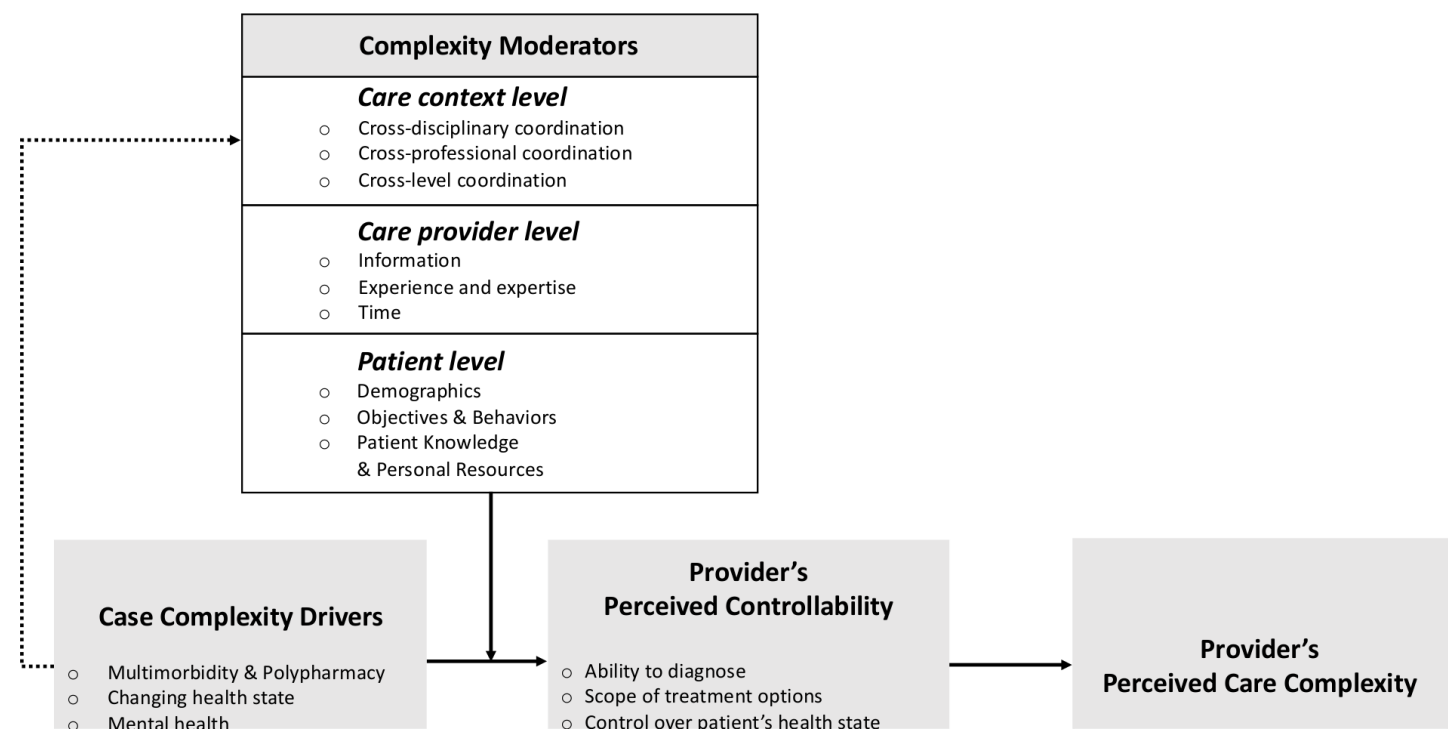

Figure 1 Perceived care complexity is driven by the provider's perceived controllability (ie, the provider's perceived ability to diagnose the patient, the assessment of the scope of available treatment options and the control over the patient's health state). Perceived controllability is driven by case complexity drivers and complexity moderators on the care context level, the care provider level and the patient level.

more complex situation, for example, would be an older patient with several unsuccessful treatments behind him. That means he will have a complex HIV treatment with maybe four, five tablets per day. Regimens that may cause complications with his kidneys or digestion or sleeping are common. And then because of his age, he may have developed other conditions, such as hypertension, which would formally have a contraindication for some HIV treatments. That means he is at risk of cardiovascular events, which we would have to check regularly with the cardiologist... So complexity entails any situation where one of his problems would influence the treatment of other problems in a negative way, such that you cannot deal with every disease optimally. (B6)

When asked what makes multimorbidity cases complex, one senior physician explained:

...whenever one starts treating an aspect of the disease, it will immediately influence other aspects. So one creates new problems and enhance complexity because another problem will pop up.... That means that when treating multimorbid patients, one has to try to anticipate what will come next in order to not miss it. With non-multimorbid patients one doesn't have to do that as much. It's much easier and takes less effort. (G1)

Participants also discussed how polypharmacy posed challenges for controlling the patient's health state:

Multimorbid patients often take a lot of other drugs. Clearly, there one has to be much more careful because of drug interactions. So the intern or I are going to spend some time on the internet platform on drug interactions in front of the patient and check if everything is okay... That takes a bit more time. (G3)

In older patients who have different conditions, many symptoms are generally caused by the drugs they are taking. Sometimes stopping or rearranging their drugs solves the problem. [But] it's difficult to spot the right moment to react. One cannot send multimorbid patients to the emergency ward every time they feel dizzy, that's not going to work... Sometimes there is a risk of missing things because one's vision is blurred by all these other problems [that] might mask something more serious. (G3)

Moreover, participants argued that they would not perceive all multimorbid patients to be complex in care delivery:

While a patient may be multimorbid, if none of the diseases are currently active but under control with whatever strategies, then it is not a highly complex situation. (B6)

Unstable or unexpected changes in patients' health conditions were another important source of complexity. As one senior nurse observed:

Complexity also arises when something new is constantly coming up. A patient with a relatively simple treatment can suddenly develop hypertension, then a heart attack, then a hip surgery. None of these have to be difficult, but it becomes complex. (C1)

\section{Mental health}

Participants discussed various ways in which patients' mental health contributed to complexity, including factors such as anxiety and depression associated with the 
knowledge of living with a stigmatised chronic disease, and other psychiatric comorbidities. A senior physician gave the following example of the complexity involved in treating a HIV patient with a psychiatric disorder:

In certain situations, patients with mental disorders go into risky behaviors that are harmful to them. We have a baseline HIV treatment, [but for these patients] I have to think about how to deal with the psychopathological condition, which may interfere with my treatment. Patients may stop taking pills and get sexually transmitted infections... [For example], when one gets a hepatitis C infection...treatment is only possible when adherence is very good. So I [have to] make sure the patient has a really good psychiatrist, and keep close contact to discuss how our treatments interact. (I2)

Mental comorbidities can also drive complexity perceptions because they hamper diagnosis. As one junior physician stated:

There are [multimorbid patients] that are very easy, and with whom it does not take a lot of time to know what the problem is. Then there are people who are so complex in their attitude. For example, patients who drink a lot may get very nervous and angry... and refuse to be examined. It can take more time to recognize other problems [in such situations]. (B1)

\section{Provider's perceived controllability}

Providers' perceived controllability refers to a provider's sense of ability to diagnose the patient, the perceived availability and scope of treatment options, and the ability to exercise those treatment options to gain control over the patient's health state. Participants noted that whether case complexity drivers would indeed translate into perceived care complexity largely depended on the extent to which such drivers limited the provider's ability to diagnose, the scope of available treatment options, and the ability to put treatment options into practice.

One senior physician discussed how multimorbidity can limit the ability to diagnose and determine the courses of intervention:

When one suspects a lung infection in an otherwise healthy person, it's mostly just a simple bacterial pneumonia. But in a multimorbid person who has kidney dysfunction, heart dysfunction, and lung dysfunction and takes multiple medications, there are many more possible reasons for lung problems. So in multimorbid patients sometimes we initially don't know the etiology. We think we have to treat the pneumonia with antibiotics, but at the same time we have to improve the kidney and heart functions. And maybe even stop the drug they have to see whether it's an infection at all. The number of possibilities multiplies.... (G4)
Another senior physician explains how his sense of perceived complexity relates to his ability to influence the patient's health state:

I would differentiate between the complexity which can be managed and the complexity which is very difficult to deal with. That is not necessarily dependent on the object of complexity. A situation can be very complex but quite easy to deal with if one has good interactions among the patient and physician and other important partners. [However,] if one has the problem that one cannot persuade the patient to take their drugs, that can be extremely difficult to manage. (I1)

In a similar vein, one senior nurse explained that:

For me, something is complex when it is difficult to find a way forward. When everything has been tried, the situation doesn't improve, and one cannot change anything. (C1)

\section{Complexity moderators: patient level}

Participants described a number of patient-related factors that enhanced or attenuated their ability to control complexity emerging from the patient's health state. These 'complexity moderators' included a patient's demographics, health objectives and behaviours, disease knowledge and personal resources.

\section{Demographics}

Age

As is well known, age increases the likelihood of multimorbidity:

Some MSMs have more sexual transmitted infections than other [patient groups]. That's something we can manage, we don't need other specialist for that.... Older patients are likely to have other problems that are not specific to HIV, like osteoporosis and high cholesterol. That's very normal but sometimes they also begin to develop forms of renal failure [or] high blood pressure...because of the HIV therapy. So we have to switch the therapy. (B7)

A senior physician explained how complexity in multimorbidity is becoming increasingly challenging in HIV clinical practice, as patients live longer due to improved therapies:

A somewhat new field for us is that we now have patients who basically live as long as patients without an HIV infection. So we have an increasing number of older patients, seventy, eighty years old, who also have many other problems.... It's not only about more pathologies and more drugs but also about psychological and social things that one wouldn't do for a young patient. Older people have trouble taking all their drugs at the right time. So one has to work more with the nurses to prepare the drugs. Providing care in a more holistic way, not only for one's specialty and the 
other diseases but also the family and social context is important in these patients. (G3)

Age was also associated with patients' ability to curtail a spiral of illnesses. As one junior nurse noted:

With older people, loneliness at home tends to come with bad nutrition, bad skin care, not drinking enough.... They take their medicine thinking: 'today the pink one and tomorrow the blue one,' more or less. Such combinations mean that people are sometimes in a very dire state when they are admitted. (C10)

\section{Sociocultural realm}

Participants also identified complexity-contributing factors in the patient's social and cultural realm. One senior physician described that:

Quite a few of our patients are migrants. There the complexity can just be a matter of language, but also understandings of health and medicine, such as the role of a physician or a nurse. These can mean very different things for somebody from Cambodia, Uganda, or Serbia... (I2)

In a similar vein, another senior physician said:

Some African patients, for example, very much fear being stigmatized if they communicate that they are HIV infected... [Or] they may go to their religious healer who says 'these drugs are bad for you' and things like that. So that is a different kind of complexity which one doesn't necessarily recognize at first. (I1)

\section{Objectives \& Behaviors}

\section{Treatment adherence and compliance}

Among the subcategories of complexity moderators, patients' adherence to treatment emerged as a particularly salient factor curtailing providers' controllability. As one senior physician explained:

The patient who comes early enough, has a preserved immune system, and no comorbidity but denies his disease and does not want to take drugs can be extremely complex to deal with. We have patients here who do not believe that they are HIV infected, or that HIV causes AIDS. These interactions can be very complicated... [After some time] one just realizes that the patient didn't take the drugs.

[In contrast], a patient who comes very late, say with a candida infection and other comorbidities, but is very willing to cooperate and to do tests, is very informed about the treatment, and tells the right things about the treatment, can be quite simple to manage because one can treat him. If the treatment doesn't work one has the right information. They are also very careful not to have drugs which could interact with their treatment so they will inform their GP and say: 'be careful, I cannot take this drug. The infectious disease physician said you have to be careful'. They come back with the list of symptoms they've had in the past three months, and a list with the drugs they did and did not take. That's a complex disease but easier to manage. (I1)

One junior physician described an example of a case in which the patient's non-adherence became a major complicating factor for care delivery:

We have a patient who has had HIV for a long time and didn't take any medication. Then he developed a lymphoma. When this was discovered he agreed to start the HIV treatment. But during routine checkups we saw that his viral load was rising... He said he was taking his HIV medication but we didn't find any drug levels in his blood, so we assume that he wasn't taking the pills, at least not regularly. Now there's a risk of developing resistance to his HIV treatment, which would mean we would have to switch to another therapy which might interfere with the ideal treatment of the lymphoma. It would cause a vicious cycle and suboptimal care of the tumor. (B6)

\section{Patient's health objectives}

Participants also noted how understanding the patient's own health objectives was important for successfully managing complex cases:

For patients, some things may be more important than [they are] for the physicians. For the physician, in general, the more acute a problem is, the more important it is. If a patient comes in with bacteraemia, bacteria grow in his blood and will kill him if we don't treat it correctly, this is the important problem for the treating physician... But if [that] patient has hip pain every day for the past ten years and will maybe have it for the next ten years, he will have hip pain as well; this bacteraemia is only a very small episode in his life. So the focus is sometimes very different. This is important information that one can get from one's patient. (G1)

In a related vein, one senior physician explained how in designing treatment plans for complex cases they

have to find solutions for multimorbid patients that are feasible at home, because they are not living in the hospital. We often forget that as physicians because we only see people as patients in a hospital. (G1)

\section{Knowledge and personal resources}

Disease knowledge and literacy

Participants explained how a patient's own understanding of their disease, and the ability to communicate their knowledge with providers moderated how the latter perceived care complexity. A senior nurse gave the following example of a patient with a high degree of literacy and knowledge about his disease: 
I noticed he had a thorough understanding of his illness. He was interested in lab results, asked questions, was very perceptive of changes and communicated those to us. I had the impression that he could assess his situation very well on his own. It helped me to understand where we stood. He had had this carcinoma for a long time, and he knew how his body functioned, so I didn't have to start from scratch... Patients who live with a chronic illness for a long time are very different to deal with than patients that don't know anything or have just heard their diagnosis. (C6)

In contrast, a junior physician noted that some patients are not receptive to information on a disease, but just want therapy:

When one tells them, 'You have a chronic hepatitis B, you're sixteen years old, it's not a problem now but it could become a problem,' they are just like 'Why don't you just give me my medication?' (B7)

A senior physician noted how the availability of online resources has increased patients' disease knowledge and literacy, and how a patient's knowledge about the disease and treatment can influence the physician's controllability:

[Patients with] chronic illnesses have had the opportunity to gather information about the disease for a long time. Often they know things even better than the physicians. I think that this is something that has changed with the availability of electronic information. An informed patient is more likely to keep on going with the treatment when problems arise, [whereas with] patients that don't understand the disease or the treatment, there is often a time where they become fed up and say, 'I've had enough, I want to go home. Please stop...' Then one has to discuss and negotiate and so on. (G1)

\section{Energy}

Within the category of personal resources, participants discussed the degree to which patients had the energy to cope with their conditions as one of the most important moderators of perceived complexity. As one senior physician notes:

Patients with multimorbidity always come to the point where they get tired, and they don't have the energy to take the next step. Then one has to try to motivate them. The psychological aspect of those treatments is important. (G1)

Yet one senior nurse explained:

Being multimorbid doesn't have to mean being limited or very ill. One can be very vital and active. There are people who come here with multiple conditions, but they seem to lead their lives and somehow manage to find a balance. (C2)

\section{Financial resources}

In comparing her interactions with multimorbid and nonmultimorbid patients, one junior physician explained the role of a patient's financial resources as follows:

When I talk to [a multimorbid] patient, I have to go through more points and ask more things about his wellbeing... what kind of social insurance or money does he have? Can he cope financially? (B5)

Discussing an example, she notes:

The goal was to have him stabilized on HIV medication so that his virus was suppressed, he wouldn't have any side effects and would feel well. And I wanted to treat his hepatitis C, but then the medical insurance said they wouldn't pay so there was nothing much we could do at that moment, and we said okay, let's put it on hold, we'll wait and maybe next year the limitations will change.

\section{Complexity moderators: care provider level}

Our findings show that providers' perceptions of care complexity were also moderated by their personal competencies and resources.

\section{Experience and expertise}

Participants repeatedly noted the critical role of experience as a key resource for dealing with complex cases. As one junior physician reflected:

The capacity to synthetise what is important and what is not, is an experience thing. I think I am doing it much better than one year ago, and I will be even better a year from now. (B7)

One senior physician explained how the source of complexity changes over time, as one gains experience with complex patients:

In the beginning one is more concerned with and focused on objective complexity, problems that the patient has. It's hard to know what to do first. Should I first treat the heart disease or the infection? One expects that if one has a plan and prescribes a treatment, the problem is solved. With time that kind of complexity gets more manageable. But [then] one recognises another type of complexity: the treatment strategy that fits patient one can be completely wrong for patient two even if they have the exact same disease. Because of the cultural environment, or because they have another understanding of the disease, or because one of them is depressed and I didn't recognize that. The interaction with the patient, the family, the culture...all these things become more important, and in the beginning, it's very hard to recognize and appreciate that. [Later, one develops] a broader view of a patient and also has these bad experiences, where one made these fantastic plans and the patient just didn't take his drugs, and one becomes incredibly disappointed. And one also develops a better 
understanding of what not to do in certain situations. [For example,] guidelines say one should screen for prostatic cancer every year, but with time one says, well this patient has other problems than screening for prostatic cancer. One has to fix other things first. And one also realises that one cannot treat patients if the psychosocial problems aren't dealt with. That's very hard to learn and difficult to train in others. (I1)

As this quote illustrates, senior physicians and nurses also showed a sensitivity to and ability to attend to "weak indicators' of complexity-referring to background indicators such as factors in the psychosocio and cultural sphere of the patient. While such factors are often expressed, they may not be readily recognised by less experienced healthcare providers.

Another senior physician noted:

The more experience I have, the more I see that simplified guidelines do not actually fit everybody. The more complex the case, the less they fit. We have to be aware of when simple guidelines don't fit the process of diagnosing and treating a patient.... One develops a feeling for when a case is more complex and needs more time for interviewing, for thinking outside of the box. (I2)

\section{Time}

Participants noted that managing care for complex patients requires considerable more time resources than for less complex patients. As a senior physician commented:

[There is a] mounting financial pressure on the health system.... [I'm concerned] that we will not be allowed to deliver the best care in complex cases because of financial restrictions. (I2)

Another senior physician discussed the repercussions of economising time on complex cases:

If one doesn't invest the time to coordinate the whole process in the beginning, one will lose more time at the end of the day because one will have to do it later anyway. (G1)

\section{Complexity moderators: care context level}

Participants identified care coordination challenges in three domains: cross-disciplinary, cross-professional and cross-level. Each of these coordination challenges influenced providers' perceived ability to control a patient's health state. Higher case complexity and higher perceived care complexity were associated with more intense coordination requirements.

\section{Cross-disciplinary coordination}

Cross-disciplinary coordination concerns the management of interdependencies across a patient's healthcare providers (eg, specialists). As one senior physician noted:
With multimorbidity there are a lot of different [specialists] involved. There is the infectious diseases specialist, the internal medicine specialist, maybe a psychiatrist, a rheumatologist.... One problem is that you have to make sure to obtain all the information from those involved. If anyone starts doing something...it will affect other problems as well. And often information gets lost because it takes time to inform each other, and not everybody does it. (G1)

Participants often described the need to organise care for complex patients across care providers, in the form of a 'care team'. As one senior researcher explains:

If one only focuses on the treatment, but skip steps in building a care team and doesn't consult with other disciplines, it won't work. Interns and junior physicians are often not very happy when one organises grand rounds, and discusses the patient's problem for hours with other physicians, because it takes a lot of time. But I try to explain to them that they really have to take this time, or the treatment will not be successful. (G1)

\section{Cross-professional coordination}

Cross-professional coordination entails managing interdependencies among practitioners in the patient's broader care environment. One senior physician explained these kinds of interdependencies with non-medical professionals as follows:

Many of our patients are in difficult social situations. It is not only communicating with other physicians but also communicating with social workers and health insurance and so on. That's an important part of the work, especially for patients who are migrants or drug users... For example, it's more difficult to have polymorbid issues dealt with when someone comes in with a translator. Prisoners come in with the police, so one has to coordinate their consultations.... These issues take a lot of our physicians' time on a daily basis. (G3)

While the need for cross-professional coordination often increases complexity, participants also noted ways in which coordination across professions helped to reduce perceived care complexity. As one junior physician explains:

Social workers provide incredible benefits. [They save] a huge amount of work. Having to fill out forms that we are not familiar with and writing letters we don't usually write [and] don't know the official formulations for, would take us double the time. (B 6)

\section{Cross-level coordination}

Some participants also identified differences in tenure and hierarchical position as a factor influencing their ability to coordinate care for complex patients. One junior 
Table 1 Perceived controllability (ie, ability to diagnose, availability and scope of treatment options; ability to exercise treatment options) of complexity-contributing factors according to provider experience level

\begin{tabular}{|c|c|c|}
\hline \multirow[b]{2}{*}{ Complexity-contributing factors } & \multicolumn{2}{|c|}{ Provider experience* } \\
\hline & Low & High \\
\hline \multicolumn{3}{|l|}{ Case complexity drivers } \\
\hline Multimorbidity and polypharmacy & Low/medium & Medium/high \\
\hline Changing health state & Low & Low \\
\hline Mental health & Low & Medium \\
\hline \multicolumn{3}{|l|}{ Patient level complexity moderators } \\
\hline Age & Low & Low \\
\hline $\begin{array}{l}\text { Managing patient's extended care } \\
\text { network (eg, family) }\end{array}$ & Low & Medium \\
\hline Cultural and language differences & Low/medium & Low/medium \\
\hline Non-adherence to treatment & Low & Low/medium \\
\hline $\begin{array}{l}\text { Diverging patient-provider health } \\
\text { objectives }\end{array}$ & Low & Low/medium \\
\hline Disease knowledge and literacy & Low/medium & Low/medium \\
\hline Energy & Medium & Medium \\
\hline Financial resources & Low/medium & Low/medium \\
\hline \multicolumn{3}{|l|}{ Care provider-level moderators } \\
\hline $\begin{array}{l}\text { Recognising weak indicators of } \\
\text { complexity }\end{array}$ & Low & High \\
\hline $\begin{array}{l}\text { Managing non-standard/non-guideline } \\
\text { cases }\end{array}$ & Low & High \\
\hline Allotting time to complex patients & Low/medium & Low/medium \\
\hline \multicolumn{3}{|l|}{ Care context level moderators } \\
\hline Cross-disciplinary coordination & Medium & High \\
\hline $\begin{array}{l}\text { Resolving cross-professional (eg, nurse- } \\
\text { doctor) information asymmetries }\end{array}$ & Low/medium & High \\
\hline Cross-level coordination & Low & High \\
\hline
\end{tabular}

*Provider experience is anchored 'low': $<3$ years postgraduation; 'high': $>3$ years postgraduation.

physician described how she initially had difficulties soliciting help from specialists outside the department:

In the beginning, I was not very confident calling other specialists. When they said they couldn't come I'd say 'ok, no problem'. That was not helpful. I learned that I had to be stricter, and approach it with more power and confidence, and more clarity about what the patient needed... Sometimes, when we leave the choice to the specialists, they'll say no. But if we say, 'You don't have a choice, you have to see this patient. It's an emergency', it works better. (B1)

Table 1 summarises the perceived controllability of complexity-contributing factors according to the provider's level of experience. The findings highlight that more experienced medical practitioners report high perceived controllability over complexity moderators at the care provider level and care context level, whereas these factors constitute important drivers of perceived patient complexity by less experienced practitioners (ie, low perceived controllability).

\section{DISCUSSION}

Since the early 1990s, global healthcare systems have come under economic pressure in the face of increased healthcare spending. ${ }^{22}$ As a result, there is an increasing demand on healthcare providers to effectively treat, document, and economise on the time spent on complex patients with high care requirements. However, what constitutes a complex patient remains poorly defined. This study sought to inform clinical practice and education in domains where patient complexity is prevalent (eg, HIV outpatient clinics) by identifying the scope and impact of patient complexity-contributing factors and the role of practitioner experience in complexity perceptions. In HIV clinical practice, improvements in antiretroviral therapies have tremendously increased the life expectancy of patients. Medical practitioners in our study explained that as HIV patients age, multimorbidity poses new challenges for managing patient complexity. Beyond providing empirical insights into specific challenges and implications for care complexity perceptions in HIV clinical practice, our study offers conceptual insights into the workings of complexity-contributing factors that may translate to the treatment of other chronic illnesses. In particular, our study advances the much-needed clarification of the concept 'complex patient'-moving from objective complexity-contributing factors relating to the patient's medical health state to a broader, holistic notion, including non-medical factors. The findings have important implications for the literature on patient complexity as well as for clinical practice and medical education.

First, this study contributes new insights into complexity drivers in clinical practice. Previous studies have provided important insights into general factors contributing to healthcare providers' perceptions of patient complexity. ${ }^{1211}$ Our study extends prior work by addressing patient complexity from the healthcare provider's perspective and conceptualising patient complexity in terms of patient characteristics and practitioner experience. As Doessing and Burau conclude in their review of the literature on multimorbidity, prior studies on care coordination for complex patients have offered little insights into the perspective of healthcare professionals in providing care for complex patients. ${ }^{14}$ Our findings elaborate prior work by identifying complexity-contributing factors and explaining the relationship among them.

Importantly, we introduce the concept of complexitymoderating factors to provide a basis for identifying the conditions that determine the extent to which case complexity translates into perceived care complexity. Case complexity drivers may not manifest in a significant way without the presence of complexity-moderating factors, which act as a 'multiplier,' akin to a coeficficient. In line with previous studies, healthcare providers in this study explained that case complexity drivers (ie, multimorbidity and interdependencies among physical and mental health state factors) generally increase the potential for perceived complexity. Yet our study participants 
also argued that complexity is only perceived as such to the extent that other patient characteristics ("case complexity moderators') and aspects of the care coordination context ('care complexity moderators' enable or constrain the controllability of the patient's health.

Our findings also foreground that the extent to which complexity-contributing factors influence providers' perceived complexity and controllability is to an important degree determined by provider experience. Understanding the role of practitioner experience in the perception of and approach to patient complexity has important implications for the development of curricula at the undergraduate and post-graduate levels, in particular in medical fields such as geriatrics and general internal medicine, in which skills for managing care for older multimorbid patients are necessary. More specifically, the findings summarised in table 1 suggest that in designing training programmes in areas where patient complexity is high, medical educators should place particular emphasis on developing skills to recognise the diverse set of indicators of patient complexity, managing non-standard/ non-guidline cases, resolving cross-professional information asymmetries and coordinating care across levels of seniority.

By conceptualising the relations among case complexity drivers, complexity moderators and perceived care complexity, our study highlights the need for designing more effective interventions and care delivery models in high-complexity healthcare settings. Medical professions are becoming increasingly specialised, while the proportion of older, more complex patients rapidly increases. Given these developments, it is critical that we understand the sources of patient complexity from the health providers' perspective before designing technological and organisational solutions that help professionals manage the cross-disciplinary and cross-professional coordination of complex cases. ${ }^{23}$

The findings and framework presented here highlight the need to develop a better understanding of the diverse drivers of complexity in healthcare training and education. As corroborated by several of our participants, there exists a need for aligning medical education with the clinical reality of managing care for complex patients. The framework developed in this study may serve as a starting point for identifying the interpersonal and coordination skills and competencies required from clinicians who provide care to complex patients in other highly complex settings such as general internal medicine and geriatrics. To advance the clinical application of our findings, table 2 presents 10 questions that may assist healthcare professionals in unravelling and communicating the nature of patient complexity. By making patient complexity more explicit, these questions can help healthcare students to more effectively identify its drivers, and develop the essential skills to manage complex patient care in daily practice.

\section{Limitations}

The results of this study have to be interpreted relative to its empirical and methodological limitations. First, our interviews focused on the complexity perceptions of practitioners working in a single setting - a university hospital's department for ID. While the physicians in our study also perform consultations for the hospital's inpatients, our interviews emphasised complexity in the ID department's treatment of outpatients. Second, the framework is grounded on data from a Swiss hospital, and should not be generalised to settings where resource restrictions are even more severe, and access to help from other specialists (eg, social workers) is more limited. Third, it is likely that these setting-specific characteristics influenced our findings. Our study includes both nurse and physicians informants. It should be noted that while the physicians included in our study work exclusively for the ID department, most of the nurses working in the ID department also work for an internal medicine outpatient clinic. Moreover, in the outpatient clinic that we studied, junior physicians have the primary responsibility over coordinating patient care. To validate our framework, further research needs to investigate other inpatient and outpatient settings. Fourth, our operationalisation of 'experience' into 'junior' or 'senior' is arguably crude. Future work should elaborate on this measure with sensitivity to the continuous nature of experience and the critical time points in a healthcare provider's career that may define it.

\section{CONCLUSION}

Studying healthcare providers in HIV clinical practice, we developed a framework explaining how a patient's case

Table 2 Complexity checklist

\section{Case complexity \\ (patient state)}

1. Medical health state: What are the patient's physical and mental comorbidities, and how may they interact in treatment?

2. Demographics: How do age, sociocultural, and economic characteristics of the patient impact diagnosis and treatment?

3. Adherence and compliance: What are the patient's health objectives and barriers to adherence and compliance?

4. Personal resources: What is the patient's level of understanding, energy and capacity for coping with disease, treatment and changes?

9. Controllability: What sources of complexity can/should I control, coordinate, delegate or defer?

\section{Care complexity} and shared? (coordinating and providing care)

5. Care team: Who are the different care actors and what are their roles and interdependencies? Are treatment roles and objectives well-defined, aligned

6. Complexity perceptions: What is complex for whom?

7. Coordination barriers: What are the potential barriers to cross-disciplinary, cross-professional and cross-level coordination?

8. Coordination tools: Which coordination and communication tools are feasible and appropriate?

10. Change: How may the sources of complexity develop in the future? 
complexity translates into a care provider's perceived care complexity. Our findings show that case complexity is moderated by the provider's sense of controllability of the patient's health state, and complexity moderators, including non-medical patient characteristics and the coordination context in which care is delivered. The framework may be used in training and educating healthcare providers with complex patient care responsibilities, and for designing future care models and interventions. Follow-up studies are needed to validate our framework in different settings, and to illuminate specific strategies and resources that providers in different professional roles use in order to manage perceived complexity.

Contributors All persons designated as authors qualify for authorship, and all those who qualify are listed; the byline includes no honorary or ghost authors. Each author has participated sufficiently in the work to take public responsibility for appropriate portions of the content. SB-M and AS contributed to study design, literature search, writing, data collection, data analysis and interpretation, and preparation of tables and figures. AH and AR contributed to data interpretation and writing. GvK and HF contributed to writing. SB-M is the guarantor. This manuscript has not been previously published and is not under consideration in the same or substantially similar form in any other journal.

Funding The authors have not declared a specific grant for this research from any funding agency in the public, commercial or not-for-profit sectors.

Competing interests AH reports grants from Viiv, grants from Gilead, grants from MSD, outside the submitted work. AR reports support to his institution for advisory boards and/or travel grants from Janssen-Cilag, MSD, Gilead Sciences, Abbvie, and Bristol-Myers Squibb, and an unrestricted research grant from Gilead Sciences. All remuneration went to his home institution and not to AR personally, and all remuneration was provided outside the submitted work. GvK reports grants from Novartis outside the submitted work.

\section{Patient consent for publication Not applicable.}

Ethics approval This study was designed and conducted with careful attention to ethical aspects, and in particular participant confidentiality. The data do not contain information about persons but about the processes in the healthcare services of the hospital. The records are anonymised. Therefore these data collection does not come under the Data Protection Act nor under the Human Research Act. These conditions render the study exempt from the requirement for ethics review board submission and approval under Swiss law, as confirmed by the Institutional Review Board of the participating institution prior to enrolling study participants.

Provenance and peer review Not commissioned; externally peer reviewed.

Data availability statement Data are available on reasonable request. Data is available from the first author upon request.

Supplemental material This content has been supplied by the author(s). It has not been vetted by BMJ Publishing Group Limited (BMJ) and may not have been peer-reviewed. Any opinions or recommendations discussed are solely those of the author(s) and are not endorsed by BMJ. BMJ disclaims all liability and responsibility arising from any reliance placed on the content. Where the content includes any translated material, BMJ does not warrant the accuracy and reliability of the translations (including but not limited to local regulations, clinical guidelines, terminology, drug names and drug dosages), and is not responsible for any error and/or omissions arising from translation and adaptation or otherwise.

Open access This is an open access article distributed in accordance with the Creative Commons Attribution Non Commercial (CC BY-NC 4.0) license, which permits others to distribute, remix, adapt, build upon this work non-commercially, and license their derivative works on different terms, provided the original work is properly cited, appropriate credit is given, any changes made indicated, and the use is non-commercial. See: http://creativecommons.org/licenses/by-nc/4.0/.

\section{ORCID iD}

Shiko Ben-Menahem http://orcid.org/0000-0002-1684-2603

\section{REFERENCES}

1 Grant RW, Ashburner JM, Hong CS, et al. Defining patient complexity from the primary care physician's perspective: a cohort study. Ann Intern Med 2011;155:797-804.

2 Loeb DF, Binswanger IA, Candrian C, et al. Primary care physician insights into a typology of the complex patient in primary care. Ann Fam Med 2015;13:451-5.

3 Loeb DF, Bayliss EA, Candrian C, et al. Primary care providers' experiences caring for complex patients in primary care: a qualitative study. BMC Fam Pract 2016;17:17-35.

4 Roosan D, Weir C, Samore M, et al. Identifying complexity in infectious diseases inpatient settings: an observation study. $J$ Biomed Inform 2017;71S:S13-21.

5 Safford MM, Allison JJ, Kiefe Cl. Patient complexity: more than comorbidity. The vector model of complexity. J Gen Intern Med 2007;22 Suppl 3:382-90.

6 Safford MM. The complexity of complex patients. J Gen Intern Med 2015;30:1724-5.

7 Islam R, Weir C, Del Fiol G, Del FG. Clinical complexity in medicine: a measurement model of task and patient complexity. Methods Inf Med 2016;55:14-22.

8 Schaink AK, Kuluski K, Lyons RF, et al. A scoping review and thematic classification of patient complexity: offering a unifying framework. J Comorb 2012;2:1-9.

9 Manning E, Gagnon M. The complex patient: a concept clarification. Nurs Health Sci 2017;19:13-21.

10 Gueler A, Moser A, Calmy A, et al. Life expectancy in HIV-positive persons in Switzerland: matched comparison with general population. AIDS 2017;31:427-36.

11 Peek CJ, Baird MA, Coleman E. Primary care for patient complexity, not only disease. Fam Syst Health 2009;27:287-302.

12 Mount JK, Massanari RM, Teachman J. Patient care complexity as perceived by primary care physicians. Fam Syst Health 2015;33:137-45.

13 de Jonge P, Huyse FJ, Stiefel FC. Case and care complexity in the medically ill. Med Clin North Am 2006;90:679-92.

14 Doessing A, Burau V. Care coordination of multimorbidity: a scoping study. J Comorb 2015;5:15-28.

15 Kvale S. The qualitative research interview - a phenomenological and a hermeneutical mode of understanding. J Phenom Psychol 1983;14:171-96.

16 Miller W, Crabtree B. Overview of qualitative research methods. In: Crabtree BF, Miller WL, eds. Doing qualitative research. Newbury Park, CA: Sage, 1992: 3-33.

17 Holstein JA, Gubrium JF. Phenomenology, ethno methodology, and interpretive practice. In: Denzin NK, Lincoln YS, eds. Handbook of qualitative research. Thousand Oaks, CA: Sage Publications, 1994: 262-72.

18 Creswell JW, Inquiry Q. Research Design: Choosing among Five Traditions. 2nd edn. Thousand Oaks, CA: Sage Publications, 2007: 38-41.

19 Smith JA, Jarman M, Osborn M. Doing interpretative phenomenological analysis. In: Murray M, Chamberlain K, eds. Qualitative Health Psychology: Theories and Methods. London: Sage Publications, 1999: 218-41.

20 Biggerstaff $D$, Thompson AR. Interpretative phenomenological analysis (ipa): a qualitative methodology of choice in healthcare research. Qual Res Psychol 2008;5:214-24.

21 Thomas DR. A general inductive approach for analyzing qualitative evaluation data. Am J Eval 2006;27:237-46.

22 OECD. Fiscal sustainability of health systems: bridging health and finance perspectives, 2015: 1-8.

23 Ben-Menahem SM, von Krogh G, Erden Z, et al. Coordinating knowledge creation in multidisciplinary teams: evidence from earlystage drug discovery. Acad Manage J 2016;59:1308-38. 